\title{
Research Contracts
}

National Cancer Institute

\section{Source}

National Cancer Institute. Research Contracts. NCI Thesaurus. Code C19004.

A research contract differs from a grant or cooperative agreement awarded in response to an RFA with regard to the rules that govern them. Contracts must comply with the provisions of the U.S. Government's Federal Acquisition Regulations \{FAR\}. The FAR includes the forms and procedures used in the solicitation for and award of contracts, and identifies the level of Government oversight of performance. 